\title{
机电一体化技术在智能制造中的实践研究
}

\author{
王卫平 \\ 江西特种电机股份有限公司，江西 宜春 336000
}

[摘要]伴随经济的迅速发展, 智能制造业在经济发展过程中所发挥的作用越来越重要, 其地位也变得分外重要, 而这样对行 业内部从业者所提出的标准和要求就更加严格, 要求其不断的提高自身的专业技术水平和综合素质才能更加准确的把握智能 制造业行业发展的特点和机电一体化技术，才能更好的促进机电一体化技术在智能制造业中得到更好的发展。 [关键词]机电一体化技术; 智能制造; 实践

DOI：10.33142/sca.v3i9.3291 中图分类号：TH39 文献标识码：A

\section{Practical Research on Mechatronics Technology in Intelligent Manufacturing}

\author{
WANG Weiping
}

Jiangxi Special Motor Co., Ltd., Yichun, Jiangxi, 336000, China

\begin{abstract}
With the rapid development of economy, intelligent manufacturing industry plays a more and more important role in the process of economic development, and its position has become particularly important. In this way, the standards and requirements put forward by practitioners in the industry are more strict, and they are required to continuously improve their professional and technical level and comprehensive quality, so as to grasp the development trend of intelligent manufacturing industry more accurately characteristics and mechatronics technology, in order to better promote mechatronics technology in intelligent manufacturing industry to get better development.
\end{abstract}

Keywords: mechatronics technology; intelligent manufacturing; practice

\section{1 机电一体化技术的智能制造概述}

机电一体化技术主要是指通过使用多种先进的专业技术来对传统的机械技术开展科学的优化和升级, 从而升级为 更为先进的技术实现对生产制造活动进行有效的引导, 实现高效率的生产, 帮助企业通过生产高质量的产生获得更高 的经济效益。机电一体化具有复杂性、紫丁花以及模板化诸多特点, 而这些特点使得其在制造业中的作用更加重要, 是促进制造业向着集约化和智能化高速发展的重要保障, 同时也使制造业的产品加工工作更加高效和规范, 生产质量 和效率都有大幅度的提高。

而智能制造业主要是在产品生产过程中在智能化技术和相关系统的基础之上来对产生的生产过程进行不断的完善 和优化, 从而通过提高工艺水平实现提高产生生产效率和生产质量的目的, 此外还能在生产过程中降低因为人为因素 而导致生产事故效率低下的问题，从而不管是生产的效率和生产的质量都得到显著的提高。

\section{2 机电一体化技术在制造业中的应用优势}

\section{1 提高产品生产质量}

传统制造业生产过程中所使用的到的机械设备一般都比较陈旧, 生产效率都比较低下, 而且整个生产过程需要依 靠大量的人力资源才能完成, 但是因为生产过程中, 操作人员对生产工序的理解不一致, 所以使得产品生产出来以后 经常会存在一定的差异, 无法满足生产标准要求, 产生了大量的资源浪费问题。但是机电一体化的使用在很大程度上 代替了一定的人力资源, 从而有效的降低了因为人为因素而导致的产品质量和管理等方面的问题, 有效的提高了产品 生产的质量, 更好的满足了生产标准的要求, 提高了生产的效率和资源利用率。

\section{2 对其他行业的发展有一定的促进作用}

在实际运用过程中, 机电一体化技术不仅能够有效的将计算机电子技术和机械技术进行有效的融合, 而且还能依 据生产的实际需要来选择更加合适的生产技术。所以伴随着制造业的飞速发展, 在生产过程中, 通过智能化技术能够 有效的将多个专业的技术进行有效的融合, 由此还能充分带动其他专业的发展。当前我国计算机电子技术的发展还处 在初级阶段, 所以导致在实际的生产过程中还不能充分实现机械设备和生产线的有效融合, 而是分开运行, 所以导致 
生产的智能化水平还比较低。但是随着电子技术的不断发展和进步, 科研人员的不断研究和实践, 从而实现了电子技 术与其他技术的结合, 由此实现了机电一体化技术, 其已经成为了智能制造业生产过程中的关键技术, 此外通过各个 专业技术能够相互弥补之间的不足, 相互促进, 在很大程度上提高了制造业智能化的水平, 不仅提高了智能制造业的 市场竞争力，而且也为制造业的长远发展提供了充分的保证。

\section{3 机电一体化技术发展历程}

很长时间以来针对机电一体化技术已经进行深入的研究和实践。在最初这种技术在使用中仅仅是被当作技术层面 的一种理念, 还没有能够将其与机械电子进行有效的融合, 所以这就导致在实际的机械制造生产过程中, 机械和电子 两个部分是独立存在的, 因此也就没有能够充分发挥机电一体化的专业优势。但是伴随计算机技术的发展和普及, 在 机电一体化技术中也逐渐了融入了该技术, 从而使得机电一体化的发展更加有活力, 也有了更多的发展发现和内容, 使其在具体的实践过程中应用的更加广泛，智能制造理念也更加被人们接受。

当前机电一体化技术包含了诸多技术, 首先就是传感技术, 该技术主要是通过在电气设备和机械设备旁边安装传

感器, 从而实现对信息进行采集和监控的目的, 如此帮助操控人员对数据进行更加全面的了解; 其次就是信息处理技 术, 通过该技术能够更加有效的增加机电信息的科学性, 而且还可以通过计算机技术对相关信息进行分析和计算, 实 现对设备参数进行调整, 实现对信息进行自动化处理的目的; 最后就是自动控制技术, 依据机械制造生产的实际要求, 来建立完善的自动化控制系统, 然后再通过 PID 闭环控制原理实现对机电设备的控制, 从而增加管理的科学性。

\section{4 机电一体化技术在智能制造中的实践要点分析}

\section{1 数控生产技术}

机电一体化技术自出现以来就被应用在机械制造业中, 并且进行了很好的运用。而在机械制造业中, 其非常重要 的一个分支就是数控生产技术，而数控生产的良好运行，必然有机电一体化技术的有效运用。

在数控生产技术运用过程中, 智能化系统的作用是非常显著的, 通过将智能化管理和数控技术进行有效结合从而 实现对相关信息的收集和处理, 从而充分保证了整个机械制造生产高效和高质的完成。当前在我国的智能数控生产技 术中, 其主要是以总主线和 CPU 为代表的, 并且得到了良好的生产和优化。例如在机械生产中, 一旦某一环节出现问 题, 智能化系统就能对其做出判断和诊断, 从而充分保证对数据进行处理和传递的效率和精确。由此可见, 在智能机 械业中, 计算机技术充分发挥了重要的作用, 并且通过对制造过程进行三维动态展示为工作人员的操作提供更多的方便。

\section{2 智能机器人}

随着智能化技术的发展, 智能机器人在制造业中出现。智能机器人的出现在更大程度上提高了生产的高效率, 同 时还保证了加工制造生产得以健康稳定的发展。比如在实际的生产过程中, 工作人员可以先对智能机器人进行任务编 程, 然后通过计算机来对其进行科学的控制, 从而充分保证了整个生产过程能够在稳定安全的环境中顺利的开展, 不 仅保证了效率, 而且还保证了生产的质量。

伴随科学技术的不断更新和发展, 科研人员逐渐的把智能机器人和智能制造进行了有效的结合, 同时对智能机器 人的各种性能进行升级和优化, 充分实现了智能机器人与人互动, 协同工作的目标。此外在一些较为特殊的生产领域 中, 因为生产过程比较危险, 比如会产生有害气体或者工作环境比较恶劣等, 如果人力操作会对人身安全产生很大的 威胁, 而这时候智能机器人就不用担心这一问题, 所以智能机器人的运用是非常必要的。

通过在机械生产中大量使用智能机器人, 使得生产力得到了解放, 而且因为智能机器人在比较恶劣的环境中也能 实施作业, 所以在很大程度上保证了生产工作的顺利开展, 同时还降低了安全事故发生的概率, 确保了企业的经济效 益, 促进其不断扩大规模, 实现更好的发展。

\section{3 传感技术}

在以往传统的生产过程中, 因为诸多因素的限制导致很多生产过程难以进行精确的操控, 而正是这一问题直接导 致了机械制造业无法真正的向着标准化的方向发展。此外, 作为机电一体化技术中的核心技术之一的传感技术, 其发 挥的作用也是至关重要的。通过传感器可以使机械制造工作具有智能化的提点, 而且通过光纤传感信号实现了过程的 自动化, 使得生产过程具有自动存储、传输以及获取的功能。在实际的应用中, 智能化的传感技术已经被应用到诸多 领域中, 比如检测声发射, 感知环境等一些比较前沿的领域, 使用过程中充分显示了其自动修复、自动适应以及识别 的强大功用, 由此可以看出, 当前传感技术的应用范围变得更加广泛, 所使用的渠道也是越来越广, 比如在汽车领域、 
医疗、消防等都得到了很好的运用，同时还实现了远程测控的功能。

\section{4 自动生产控制技术}

通过对机电一体化自动生产控制技术的研究不断深入, 由此对加工制造产品的生产过程了解的更加充分, 从而有 效的避免因为人为因素而导致的各种质量问题出现, 因为通过机电一体化技术能够在生产过程中及时发现其中存在的 问题, 并且及时的对其进行处理, 由此充分保证了生产活动的顺利开展。此外就是通过近些年的相关文献发现, 自动 化生产过程其不管是实用性还是先进性都具有很大的优势, 而且自动生产过程中的跟踪系统不仅能对生产全过程进行 全面充分的管控, 同时还能对整个生产过程中产生的数据进行收集、分析与处理, 通过事先设定好的程序和标准化生 产为企业的顺利开展提供看可靠的保证。

\section{5 柔性控制系统}

通过使用柔性控制系统, 能够依据生产的实际情况开展数字和信息的控制功能, 同时还可以实现自由的转换, 依 据该技术, 能够将生产过程中所需要的设备和物料等资源实现智能化的关, 多个生产线可以同时开展工作。再有就是 通过信息技术对市场数据进行全方面的分析, 由此对市场的发展态势进行充分的了解, 并在此基础之上对原来的生产 计划进行科学的调整, 避免出现资源浪费的情况。此外通过柔性控制系统还能对相关的物流信息系统或者自动加工系 统来对现有的生产系统进行科学的调整, 从而实现生产优化的目的。

\section{5 结语}

总之, 通过在智能制造生产中使用机电一体化技术能够使其发展前景更加广阔。机电一体化技术的运用不仅能够 提高生产的效率和产品的质量, 同时还能节省企业各种资源, 降低生产施工成本, 从而帮助企业拥有更多的资源去发 展, 从而获得更高的经济效益和社会效益。但是当前机电一体化技术在智能制造中的应用还有很大的发展潜力, 需要 对其进行更加深入的研究，寻找更加适合的发展渠道，促进我国制造业向着更高更强发展。

\section{[参考文献]}

[1]刘金涛,翟昱尧. 机电一体化技术在智能制造中的发展与应用 [J]. 科技创新与应用, 2020 (18): 174-175.

[2]孙玉强. 机电一体化技术在智能制造中的实践研究 [J]. 智能城市, 2020 (11) : 243-244.

[3]李雨菲,刘雅文. 机电一体化技术在智能制造中的应用 [J]. 数码世界, 2020(4): 281 .

[4]何晓婵.机电一体化技术在智能制造中的运用实践 [J]. 产业创新研究, 2020(22) : 150-151.

作者简介: 王卫平 (1969-) 男, 江西江特电机有限公司, 研发中心, 总工程师/专家, 高级工程师, 专业: 机电一体 化, 电机研发设计。 